\title{
Experimental Performance Testing of APA Stack Actuator for a 2 DOF Microgripper
}

\author{
Royson Donate D’Souza
}

\begin{abstract}
This paper presents the experimental testing and results of Amplified Piezoelectric Actuator (APA) 120S which is used as an actuator for a 2-DOF compliant microgripper. APA is characterized using a unique static force measurement technique, to get the graphical relationship of Force-Displacement-Voltage which can be used determine the input force to the microgripper. Further this force is compared with the real time force measured using HBM force sensor on the microgripper test setup. An average deviation of $9.74 \%$ is observed from the real time measured values and $10.33 \%$ deviation from the FEM results. A gripping force of $1.8 \mathrm{~N}$ is observed for the input voltage of $140 \mathrm{~V}$ to the APA $140 \mathrm{~S}$ actuator. Experimental measured values provide a good validation for the theoretical values.
\end{abstract}

Keywords - 2 DOF microgripper, Amplified Piezoelectric Stack Actuator (APA), Strain gauge, cantilever beam force sensing.

\section{INTRODUCTION}

Microsystem technology or MEMS has drastically increased the modality of sensing and actuating techniques leading to a demand and increase in MEMS devices. Assembly of microsystems still forms a challenging task to the researchers. Micro gripper is one of the key tools to realize the micromanipulation and micro assembly of micro objects.

Several types of microgrippers are developed in the past decade using different types of actuating principles like Electrostatic microgripper [1]-[2], Electrothermal microgripper [3]-[4], Shape memory alloy actuated microgripper [5]-[7], Piezoelectric based microgripper [8]-[10]. Piezoelectric materials have large operating bandwidth and can produce large mechanical forces in a compact design which makes it more feasible and befitting solution for the microgripper actuators. Microassembly being the major concern number of degrees of freedom (DOF) play a very important role. Microgripper is said to have have single DOF if it can only grasp an object [11] and a 2 DOF if it could grasp and rotate [12]- [13]. 2 DOF microgripper find its applications in microrobotics and placing optical fibres in MEMS opto switches [12].

Characterising the actuator forms a basic itenary task for the actuation of the microgripper. Due to the three variable parameters Force, displacement and Volatge which play a

Manuscript received Nov 18, 2015. UAE.

Royson Dsouza is with Manipal School of Engineering and IT, Dubai campus, mojor role in the precise control of the actuator, need for characterising the actuator to get a graph of Force, displacement and Volatge is very essential. Many researchers have provided various techniques for the characterization of the Piezoelectric actuators. Force and displacement at various voltages are determined using the method of incremental loading [14]. The method combines an actuator input voltage and a current together with a displacement measurement to a force estimator. Experimental performance of small , medium and large scale piezoelectric actuators are tested by compressing the actuators and measuring the forces via load cell [15]. This method is more applicable to measure large actuation forces. Analyses of blocking force of Piezoelectric stack actuators PXE5, PC5H and Px28 is carried out at low electric field [16]. The results are compared with Finite Element Method [FEM] having good correlation with the values. A detailed theorotical modelling and experimental validation is carried out for the piezoelectric actuator of type Jena P-177-00 large stroke piezoelectric actuator [17]. The test setup is both suitable for static and low frequency dynamic testing of the Piezoelectric stack actuators. The experimental test setup was based on the principle of compression of Proving ring at various input voltages and corresponding force is measured using a Force dial guage. The tests are carried out under static conditions of the piezoelectric stack actuators proving two unique methods of obtaining Force-Displacement-Voltage relationships.

Experimental setup integrating electrical and mechanical setups based on a $\mathrm{T}$ shaped console is used to investigate the force and displacement of the Piezoelectric stack actuators [18]. An experimental study on displacement self sensing method is studied to obtain output displacement signals of piezoelectric stack actuators [19]. A good correlation between the theorotical and experimental value is observed. A detailed experimental setup to evaluate the performance of various types of piezoelectric actuators is developed and tested for both AC and DC input signals [20]. The Piezoelectric stacks were tested at extreme voltages and temperatures and at various frequency conditions. This test provided the experimental results for displacement, impedance, capacitance and leakage current. A low cost adjustable experimental setup is developed to explain the performance of the actuator part of the Piezoelectric generator [21]. Overall a simple experimental setup which will caterer the need of the experimental value of force-voltage-displacement is the major interest which will be presented in this paper.

A 2 DOF microgripper is designed [13], fabricated and 
experiments are to be conducted on the same. Figure 1 shows the configuration of the designed microgripper which will taken for study. The designed microgripper can grasp and rotate the microobjects. Maximum input force calculated for the right jaw using analytical method is $22.3 \mathrm{~N}$ and left jaw is 12.08 N. Based on the FEM results and analytical results APA $120 \mathrm{~S}$ is the best suited Piezoelectric Stack actuator from the manufacturer CEDRAT TECHNOLOGIES ${ }^{\circledR}$.

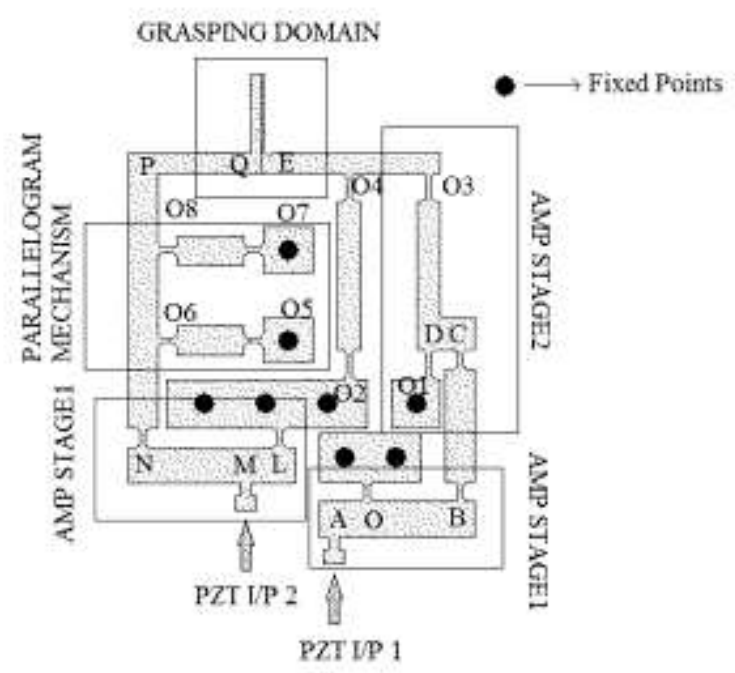

Fig. 1: Microgripper design and configuration

An experimental setup based on the cantilever beam force sensing principle is employed to measure the force and displacement characteristics of the Piezoelectric stack actuator APA 120S. Several tests are conducted to plot the relationship of Force-Voltage for the input voltage range of -20 to $160 \mathrm{~V}$ to the APA 120 S stack actuator. Later the microgripper is put under test providing the input from the Piezoelectric actuator and corresponding force is calculated using $\mathrm{HBM}^{\circledR}$ force sensor U9C. The values are compared with the FEM results. A simple and a precise technique to evaluate the Force-Displacement-Voltage characteristics of APA $120 \mathrm{~S}$ piezoelectric stack actuator is presented in this paper.

\section{TESTING OF ACTUATOR}

APA 120S is the Amplified Piezoelectric actuator under study. The datasheet provided by the manufacturer CEDRAT TECHNOLOGIES $^{\circledR}$ provides the extreme values for the actuator. Hence it is very essential to obtain the intermediate and detailed values of Force and Displacement for the set of input voltages. Therefore a simple experimental setup based on the strain and displacement of the cantilever beam is devised.

\section{A. Cantilever beam design}

Based on the study, cantilever based strain gauge technique [14], [18] is the most suitable testing method for experimental evaluation of the relationship of force-displacement-voltage.

The deflection at a specific point for the cantilever beam is given by

$$
y=\frac{P X^{2}}{6 E I}(3 L-X)
$$

Where $\mathrm{P}$ is the $\operatorname{Load}(\mathrm{N}), \mathrm{I}$ is the moment of Inertia $\left(\mathrm{m}^{4}\right), \mathrm{X}$ is the effective length $(\mathrm{m}), \mathrm{L}$ is the total length $(\mathrm{m}), \mathrm{E}$ is the modulus of elasticity $\left(\mathrm{N} / \mathrm{m}^{2}\right)$.

Stiffness $\mathrm{k}(\mathrm{N} / \mathrm{m})$ of the cantilever beam is given by,

$k=\frac{3 E I}{L^{3}}$

Knowing the above parameters and calculating the displacement of the cantilever beam, Force can be measured using equation (3),

$F=k x$

Other way of calculating the force is by measuring the strain and then relating it with the input force.

$$
\varepsilon=\frac{z P(L-X)}{E I}
$$

where ' $\varepsilon$ ' is the axial strain at a point ' $x$ ' distance from the fixed support and ' $z$ ' distance away from the neutral axis for a force ' $P$ '.

The most integral part of the test setup forms the design and optimization of the cantilever beam. Graphical optimization technique is used to optimize the dimensions of the cantilever beam for the required specifications. Using Graphical optimization technique the dimensions of the cantilever beam are decided. The objective functions are derived to be

$$
\begin{aligned}
& g_{1}(x)=\frac{6 \cdot F \cdot x \cdot F O S}{\sigma_{y} d^{2}} \\
& g_{2}(x)=\frac{4 \cdot F \cdot x^{3}}{E t d^{3}}
\end{aligned}
$$

The geometric constraints are taken as the length and width of the beam which are detailed in Table 1. Plotting the objective functions and geometric constraints using MATLAB $^{\circledR}$ as shown in Fig.2, the optimized length and width of the beam is obtained. A cantilever beam with optimized values is designed and analyzed using a FEM package $\mathrm{COMSOL}^{\circledR}$. L shaped bracket is designed, replicating a real time model analysis and the total assembly is tested for its deformation and stress analysis as shown in Fig. 3.

\begin{tabular}{|c|c|} 
TABLE I: CONSTRAINT PARAMETERS FOR OPTIMIZATION \\
\hline Design Variable & Value \\
\hline Maximum Applicable Force (F) & $60 \mathrm{~N}$ \\
\hline Factor of Safety (FOS) & 1.5 \\
\hline Young's Modulus for Al (E) & $70 \mathrm{GPa}$ \\
\hline Yield Stress for Al $\left(\boldsymbol{\sigma}_{y}\right)$ & $150 \mathrm{MPa}$ \\
\hline Max Allowable Deflection $(\mathrm{d})$ & $1 \mathrm{~mm}$ \\
\hline
\end{tabular}




\begin{tabular}{|c|c|}
\hline Thickness of beam (t) & $3 \mathrm{~mm}$ \\
\hline Geometric Constraints & Feasibility Region \\
\hline Length of Cantilever Beam (L) & $40 \mathrm{~mm}<\mathrm{L}<150 \mathrm{~mm}$ \\
\hline Width of Cantilever Beam (W) & $10 \mathrm{~mm}<\mathrm{W}<25 \mathrm{~mm}$ \\
\hline
\end{tabular}

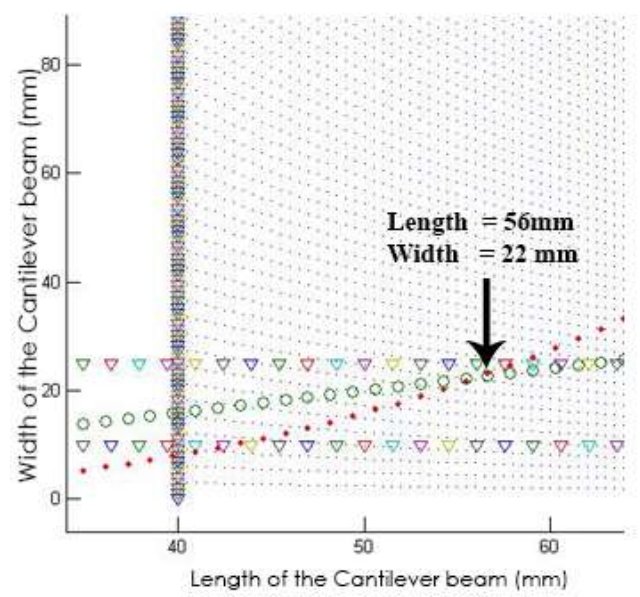

Fig. 2: Optimization graph of cantilever beam design

From the FEM results, the maximum stress is found to be 100.52Mpa which is within the limit of the design parameter. The displacement value is found to be well in correlation with the theoretical values.

\section{B. Construction of the experimental setup using L-shape module:}

The main of the experimental setup is to actuate the APA $120 \mathrm{~S}$ actuator at different voltages and different loading conditions and determine the displacement respectively in the quasi static conditions. Based on which an experimental setup is designed as shown in Fig. 4.

Fig. 4 depicts the mechanical setup to evaluate the performance of the APA120S stack actuator. The cantilever beam along with the $\mathrm{L}$ shape bracket is made to slide along a cylindrical structure which is fixed to the base. Optimum location to measure stain is located and strain gauge is embedded in to the beam.
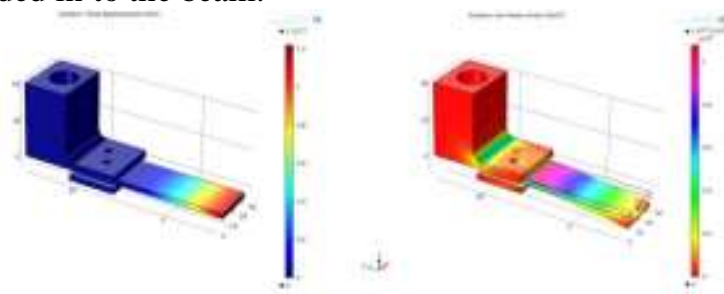

Fig. 3: Displacement and Stress distribution of L shaped module

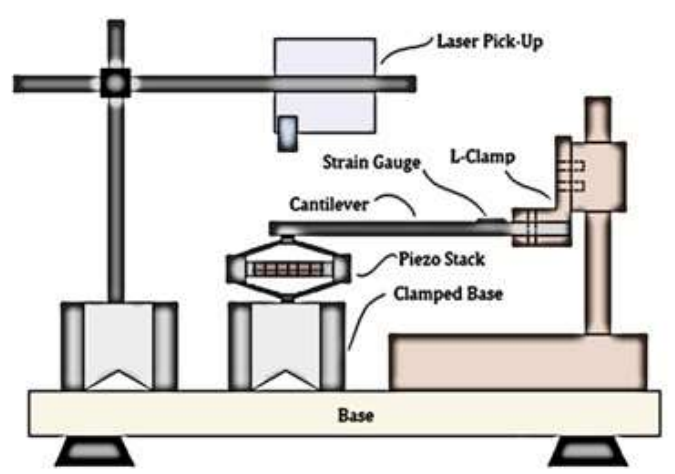

Fig. 4: Schematic diagram of experimental setup

The actuator is attached to a cantilever beam tip and the block using M2 bolts. OPTO NCDT- 1402 laser pickup from the manufacturer Microepsilon ${ }^{\circledR}$ having a resolution of $1 \mu \mathrm{m}$ is used. The entire setup is mounted on a cast iron plate seated with rubber bushings.

The strain gauge is connected to NI-DAQ 9237 via breakout board, which is embedded with a built-in half/full Wheatstone bridge. The input excitation voltage is provided by NI-DAQ 9264 which is further amplified by CEDRAT Amplifier LA-75 having a gain of 20. The electrical block diagram is shown in Fig. 5. Data acquisition and processing is carried on using LabVIEW $^{\circledR}$ software.

The photograph of experimental setup is shown in Fig. 6. The detailed view of laser reflecting off the screw head and the strain gauge location is shown in Fig. 7. Initially to verify and test the force sensing setup, standard weights are placed on the top of the beam in the optimum location. To ensure steady readings moving average is used in LabVIEW software, so that the readings are steady even at varying conditions.

\section{THE EXPERIMENTAL PROCEDURE}

Initially the displacement of the piezo-actuator APA $120 \mathrm{~S}$ is measured in blocked-free condition for the voltage change in the steps of $10 \mathrm{~V}$. The maximum displacement of $118 \mu \mathrm{m}$ is observed as shown in Fig. 8 which is in good agreement with the datasheet. As shown in Fig. 8 a small value of hysteresis is observed which can be compensated using a closed loop control system.

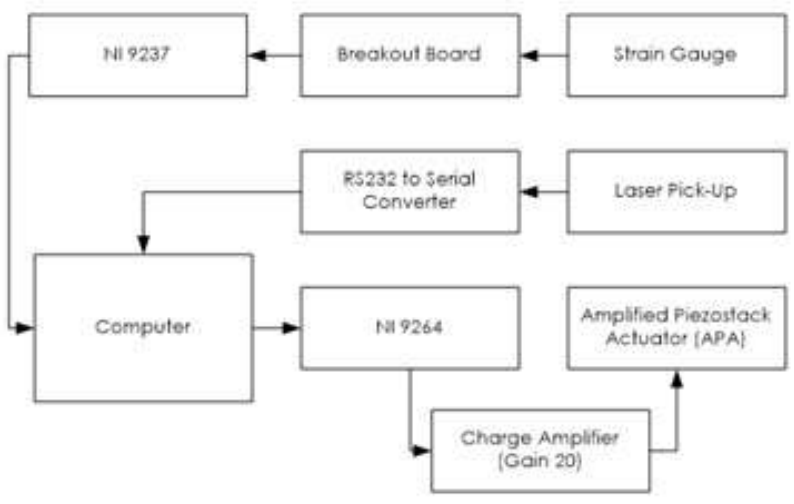

Fig. 5: Wiring block diagram of the experimental setup 

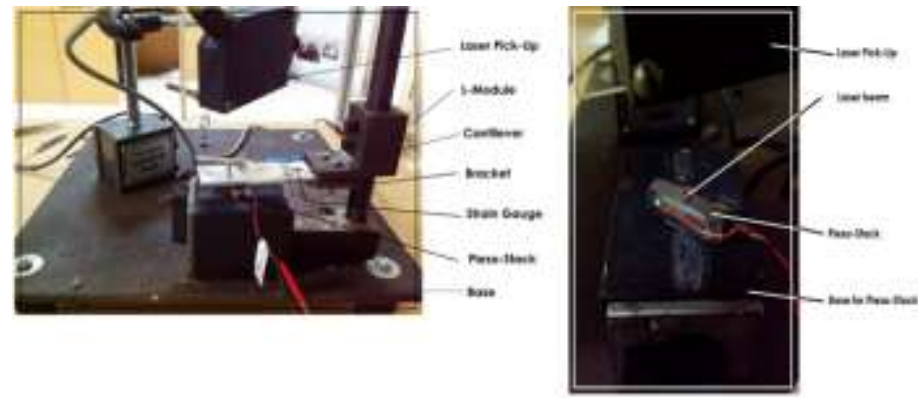

Fig. 6: Experimental setup for testing APA $120 \mathrm{~S}$

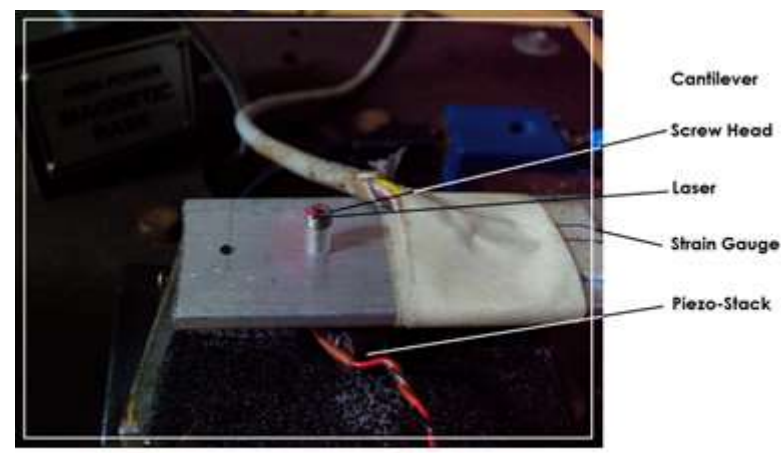

Fig. 7: Detailed view of cantilever beam used in the setup

To study the behavioral characteristics of force-displacement-voltage relations of APA 120S, a experimental procedure is conducted and the data points are recorded using two different procedures. This is due to the fact that the data points lie below and above the external stiffness curve of the cantilever beam. For the data points lying above the external stiffness curve the procedure is given as follows:

1. Actuator APA 120S is fixed to the cantilever beam with screw and slightly is pre-loaded up to $1 \mathrm{~N}$.

2. Compress the actuator upto a desired pre-stress level $F_{i}$ by sliding the cantilever beam assembly and measure the change in the displacement.

3. Apply voltage $\mathrm{V}_{\mathrm{i}}(\mathrm{i}=0 \mathrm{~V}$ to $160 \mathrm{~V})$ to the stack and record the values of force and displacement.

4. Repeat steps 1 to 3 for the next initial level of pre-stress $F_{i}$ $+1>F_{i}$

For the data points lying below the external stiffness curve, the following procedure was used:

1. Completely discharge the stack actuator.

2. Apply the voltage $\mathrm{V}_{\mathrm{i}}$ on the unloaded stack (Stack is not connected with the cantilever beam).

3. Actuator APA $120 \mathrm{~S}$ is fixed to the cantilever beam with screw and slightly compressed up to $1 \mathrm{~N}$ to ensure the contact.

4. Apply the voltage $V_{k}>V_{i}$ and record the force and the displacement from the Labview panel.

5. Repeat steps 1 to 4 for the rest of the voltages up to the maximum voltage.

Further these data points are plotted to obtain a graph of Force-Displacement-Voltage.

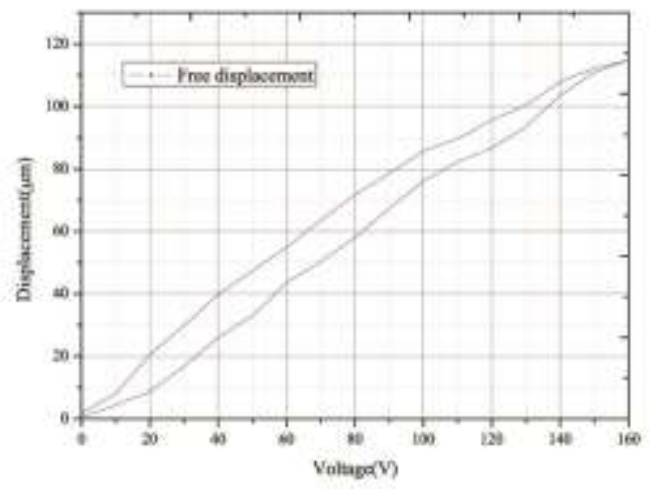

Fig. 8. Free displacement curve for APA- 120S

The Fig.9 describes the behavior of APA 120S obtained from the experiment. The plot consists of three variables i.e., force, displacement and voltage. Using any two values from the graph a third value can be easily predicted.

\section{EXPERIMENTAL SETUP TO EVALUATE THE INPUT FORCE TO THE MICROGRIPPER}

One of the unique ways of determining the input force to the microgripper using Piezoelectric actuator is discussed in the previous section using a cantilever beam and subsequent plot for Displacement-Force-Voltage for APA 120 S is been plotted. The results need to be validated by testing it on the microgripper. This will ensure the correctness of the experiment of the pre-determined force and also will provide a platform to validate the predicted results. The setup is designed to accommodate two APA-120S piezo actuators, one for each axis, force sensor, Amplifiers for the stack actuators and force sensor.

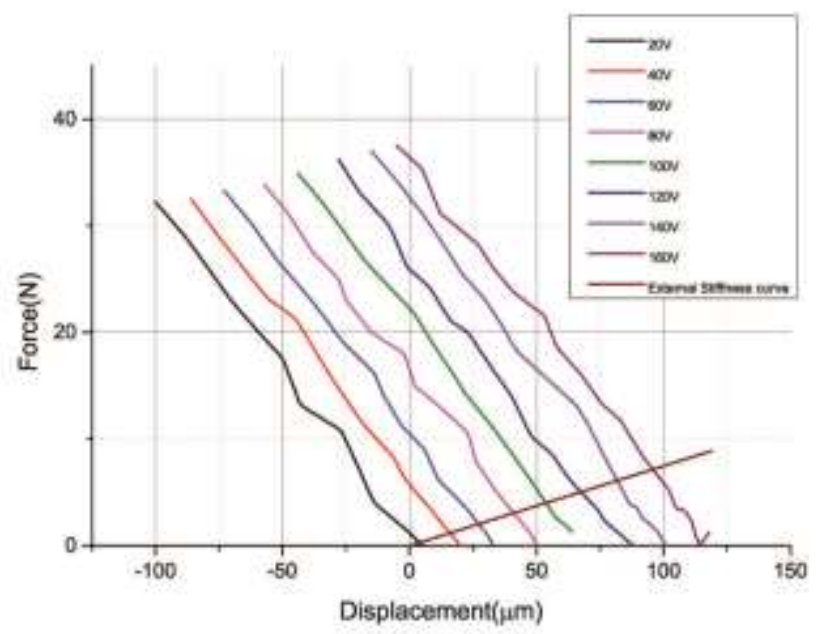

Fig. 9: APA 120S behavior described by the force-displacement-voltage correlation 


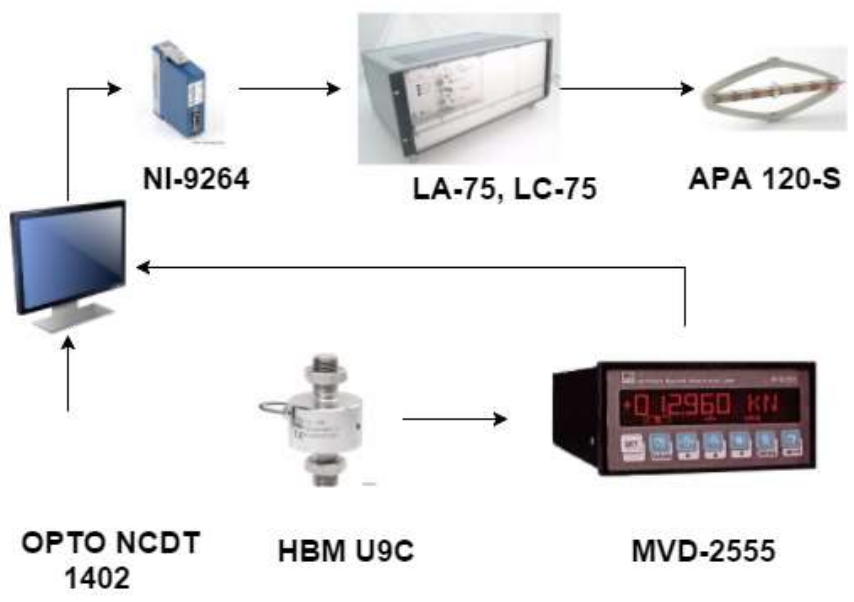

Fig. 10: Block diagram of the experimental setup

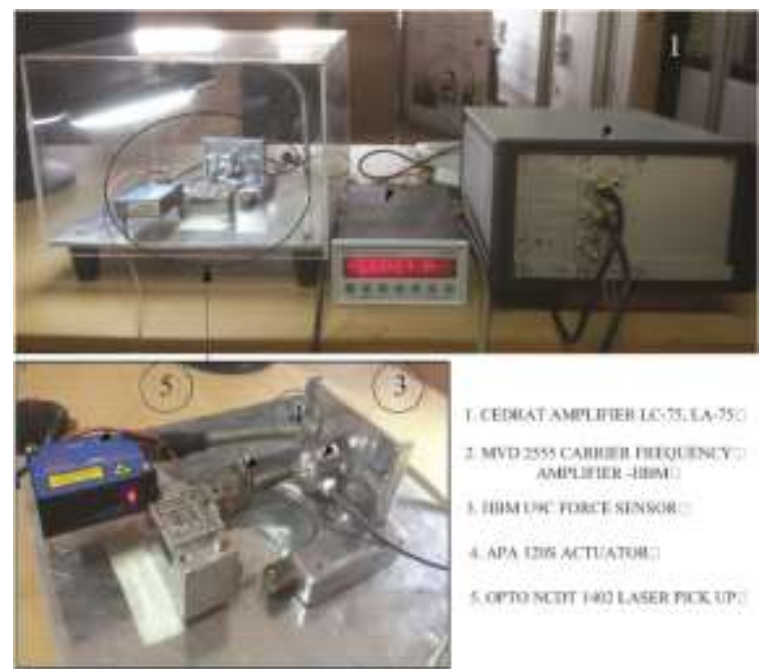

Fig. 11: Experimental setup to evaluate the performance of the APA $120 \mathrm{~S}$

Fig. 10 illustrates the block diagram of the instrumentation used in the setup. Fig. 11 details out the experimental setup developed to test the input force to the microgripper. The setup consists of APA-120S Piezoelectric working in the voltage range of $-20 \mathrm{~V}$ to $150 \mathrm{~V}$. LC-75 is a bipolar Linear AC/DC converter used to produce stabilized DC voltages to the amplifier with the current limitation of $0.12 \mathrm{~A}$. LA-75 is a linear amplifier to drive the piezo actuator APA $120 \mathrm{~S}$ with the gain of 20. It is a low noise, low power amplifier with three independent channels. Two channels are been used for right and left jaw respectively. The output of the LA75 is fed to the piezoelectric actuator. The input voltage range is fed from NI-9264, an analog output module from National Instruments and is precisely controlled using Labview.

A Carrier Frequency amplifier MVD-2555(Make: HBM) is used to measure the force which is particularly stable and insensitive to interference sensed by the HBM U9C force transducer. The measured values are read over the LCD display.

U9C series transducers are strain gauge based transducers which can be actively used for both Static and Dynamic conditions. Since the measurements are static they are best suited for the experiment. To measure output displacement of the microgripper a high resolution laser pick up is used. OPTO NCDT 1402 from Micro-Epsilon having a resolution of $1 \mu \mathrm{m}$ is used for this purpose.

NI9264 DAQ is a 16 channel analog output voltage DAQ used to supply Voltage in the range of $-10 \mathrm{~V}$ to $10 \mathrm{~V}$. This analog output voltage is given to the ac to dc converter LC-75. The Linear DC voltage is then amplified by the linear amplifier LA-75. This output is directly fed to the piezoelectric stack actuators. The actuators were actuated individually and the output displacement of the stack is recorded. On the other hand the HBM force transducer is connected with one side fixed to the guide-way and the other end to the actuator. The guideway is locked so that the motion is restrained. For the given voltage input force to the microgripper is recorded. The entire experimental setup is enclosed in a box to avoid noise and interference.

\section{TEST RESULTS OF INPUT FORCE TO THE MICROGRIPPER}

Input force to the microgripper forms a very important parameter in evaluating the performance of the microgripper. The preliminary experiments results performed using the cantilever beam for APA 120 S as detailed in previous sections, provides us the graph indicating the Force Vs Displacement at various levels of input voltages provided to the stack actuator. Input voltage and the input displacement can be easily obtained from the microgripper test setup using NI 9264 and laser pickup respectively. If these values are plotted in the graph of Force Vs Displacement at various steps of voltages we can obtain a three dimensional graph wherein the third dimension indicates the force, which is the predicted force as shown in Fig. 13.

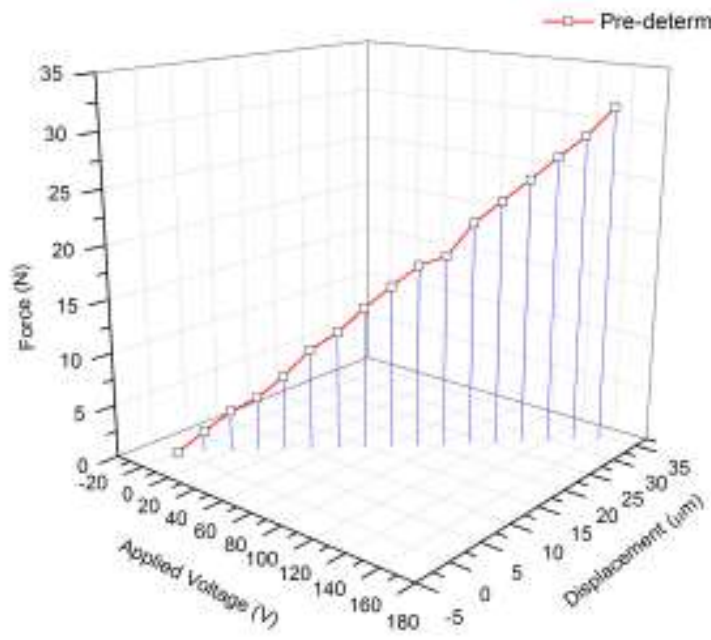

Fig. 12: 3D plot of predicted force using the experimental data of the APA120S.

To validate this result, force sensor is incorporated with the microgripper and the experiment gives us the input force to the microgripper which is further compared to the predicted experimental results. A 3D plot of Displacement-Force-Voltage is plotted based on the experimental results as shown in Fig. 14. A maximum of 29.07 
$\mathrm{N}$ force is measured on the input side of the microgripper for the input force of $34 \mu \mathrm{m}$ as shown in Fig. 15.

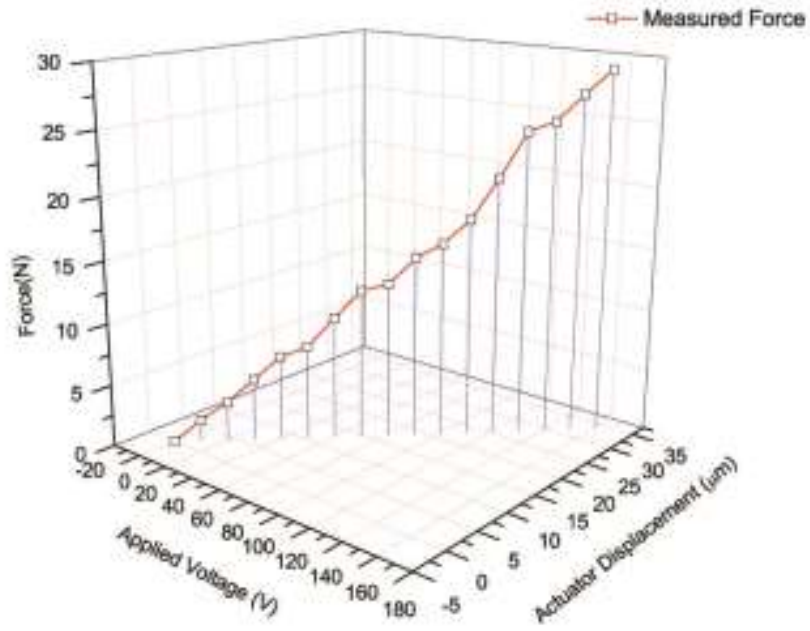

Fig. 13: 3D plot of measured force using the experimental data of the force sensor.

For further analysis the Displacement-Force plots of the measured force, predicted force and FEM (ANSYS) are plotted as shown in the Fig. 16.

The plots clearly indicate that there is a very small deviation in the measured force and the predicted force. Fig. 17 shows the percentage of error of the measured force using force sensor from the pre-determined force.

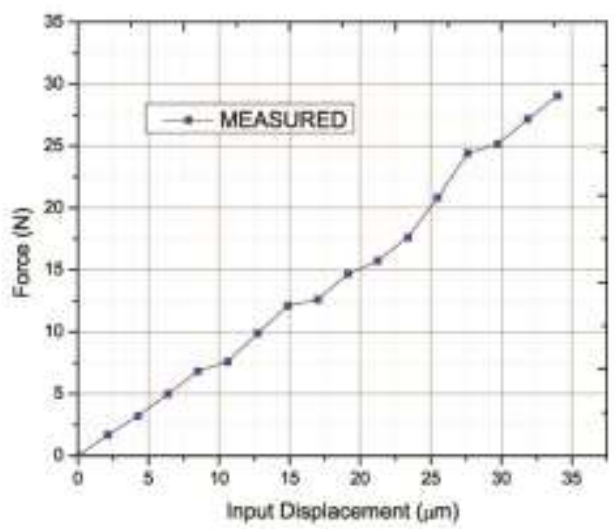

Fig. 14: Plot of measured values of Force and Input displacement.

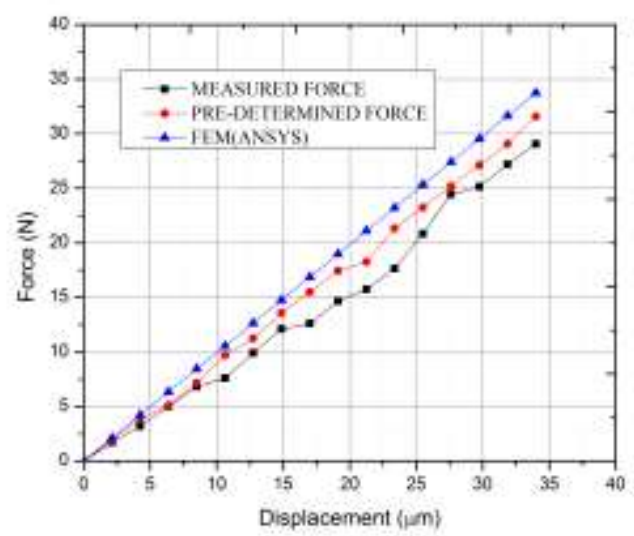

Fig. 15: Plot of Comparison of force parameters.

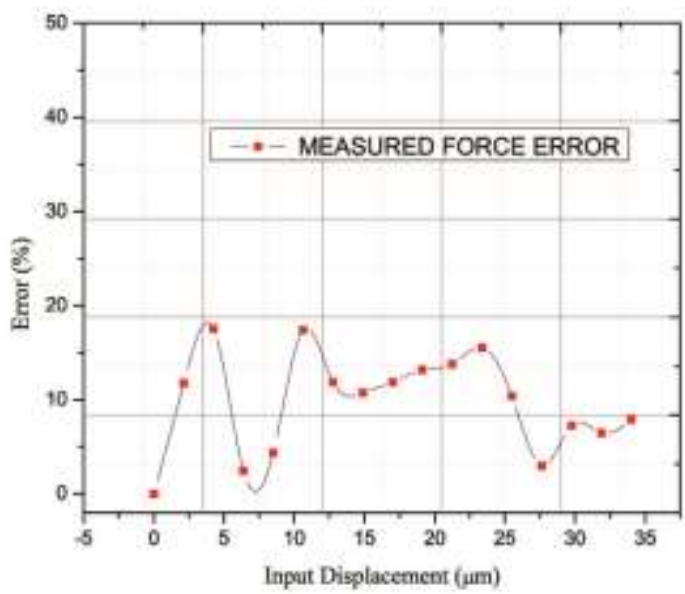

Fig. 16: Plot of loss factor Vs Input displacement of the microgripper

\section{CONCLUSION}

From the preliminary tests and experiment it can be concluded that,

- An average error of $9.743 \%$ in the measured force is observed as against the predicted force.

- An average error of $10.33 \%$ in the measured force is observed when compared to the FEM force analysis.

- Since the measured force tends to the optimal value of the pre-determined force, one can easily predict the applied force on the microgripper using the pre-determined force experiment.

- The method of pre-determined using cantilever beam experiment can be extended to other Piezo stack actuators and find their FORCE- VOLTAGE- DISPLACEMENT relation and its influence on the external structure.

\section{ACKNOWLEDGMENT}

The authors acknowledge the funding support from SOLVE: The Virtual Lab @ NITK (Grant number:No.F.16-35/2009-DL Ministry of Human Resources Development) (www.solve.nitk.ac.in) and experimental facility provided by Centre for System Design (CSD): A Centre of excellence at 
NITK-Surathkal. The authors acknowledge the helpful discussions and guidance from Dr. K. V. Gangadharan, Professor, Department of Mechanical Engineering, NITK-Surathkal.

\section{REFERENCES}

[1] K. Amjad, S. A. Bazaz and Y. Lai, "Design of electrostatic MEMS microgripper system integrated with force sensor," International conference on, 2008. http://dx.doi.org/10.1109/icm.2008.5393544

[2] M. Boudaoud and Y. Haddab, "Modeling and optimal force control," IEEE/ASME transactions on mechatronics, vol. 18, no. 3, 2013. http://dx.doi.org/10.1109/TMECH.2012.2197216

[3] N. Ali, R. I. Shakoor and M. M. Hassan, "Design, Modelling and silulation of Electrothermally actuated Microgripper with capacitive contact sensor," IEEE, 2011.

[4] R. Zhang, J. chu, H. Wang and Z. Zen, "A multipurpose electrothermal microgripper for biological micromanipulation," Microsystems Technologies, vol. 19, pp. 89-97, 2013. http://dx.doi.org/10.1007/s00542-012-1567-0

[5] Kyung, J. H. Ko, B. G. Ha, Y. H and C. G. J, "Design of a microgripper for micromanipulation of microcomponents using SMA wires and flexible hinges," Sensor. Actuat. A, pp. 144-150, 2007.

[6] A. P. Lee, D. R. Ciarlo, P. A. K, Krulevitch, S. L and J. M. Trivino, "A practical microgripper by fine alignment, eutectic bonding and SMA actuation," Sensors , pp. 755-759, 1996.

[7] K. Houston, C. Eder, A. Seiber, A. Menciassi, M. C. Carrozza and P. Dario, "Polymer sensorized microgripper using SMA actuation," IEEE International conference on Robotics and Automation, 2007.

[8] Y. Haddab, N. Chaillet and A. Bourjault, "A microgripper using smart piezoelectric actuators," Proceedings of IEEE/RSJ International Conference on Intelligent Robot and Systems Takamatsu - Japan, 2000. http://dx.doi.org/10.1109/iros.2000.894679

[9] X. Huang, J. Cai, M. Wang and X. Lv, "A piezoelectric bimorph microgripper with micro force sensing," Proceedings of IEEE international conference on Information Acquisition, 2005.

[10] D. H. Kim, B. Kim and H. Kang, "Development of Piezoelectric polymer based sensorized microgripper for microassemby and micromanipulation," Microsystem Technologies 10, 2004.

[11] Kemper, M., Development of a tactile low-cost microgripper with integrated force sensor. In Control Applications, 2004. Proceedings of the 2004 IEEE International Conference on, volume 2. IEEE, 2004 http://dx.doi.org/10.1109/cca.2004.1387581

[12] Shi, X., W. Chen, J. Zhang, and W. Chen, Design, modeling, and simulation of a 2-dof microgripper for grasping and rotating of optical fibers. In Advanced Intelligent Mechatronics (AIM), 2013 IEEE/ASME International Conference on. IEEE, 2013

[13] D'souza, Royson, Suraj Kumar, and Navin P. Karanth. "Design, Modeling and Simulation of a 2-DOF Microgripper using Piezoelectric Actuator." Recent Trends in Electronics \& Communication Systems 2.1 (2015): 10-18.

[14] P. Ronkanen and H. Koivo, 'Simultaneous Actuation and Force Estimation Using Piezoelectric Actuators', in International Conference on Mechatronics and Automation, Harbin, China, 2007, pp. 3261-3265. http://dx.doi.org/10.1109/icma.2007.4304084

[15] M. Shimazaki and T. Fujita, "Experimental study of piezoelectric actuators and magnetostrictive actuators for large-scale smart structures " 14th world conference on earthquake engineering, 2008

[16] Mekid, S., and B. Lim. "Characteristics comparison of piezoelectric actuators at low electric field: analysis of strain and blocking force." Smart materials and structures 13.5 (2004): N93.

[17] Giurgiutiu, Victor, and Radu O. Pomirleanu. "Modeling and characterization of piezoelectric and magnetostrictive induced-strain actuators." SPIE's 8th Annual International Symposium on Smart Structures and Materials. International Society for Optics and Photonics, 2001.

[18] Marinov, Mihail. "Experimental Set-Up for Complex Investigation of Stack Piezo-Actuators."

[19] Zhang, Zhonghua, et al. "Experimental Study on Displacement Self-Sensing Method Based on Multiple Piezoelectric Effects for Microactuators." Advances in Information Sciences \& Service Sciences 4.6 (2012).
[20] Sherrit, Stewart, et al. "Multilayer piezoelectric stack actuator characterization."The 15th International Symposium on: Smart Structures and Materials \& Nondestructive Evaluation and Health Monitoring. International Society for Optics and Photonics, 2008.

[21] SNS, Jamaludin, and Abu Bakar Rosli. "Experimental Performance Testing Of The Actuation Part Of Piezoelectric Generator For Alternative Power Generation." (2010).

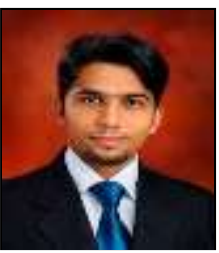

Royson Donate D'Souza received the M.Tech (Research) degree in Design and Precision Engineering from National Institute of Technology Karnataka (NITK), India in 2015 and B. E degree from Canara Engineering College, Mangalore, India in 2011. His primary research areas lie in the emerging fields of MEMS, Mechatronics and Compliant Mechanisms.

Royson Dsouza is currently working as Assistant Professor at Manipal School of Engineering and IT, Dubai Campus, UAE. 